\title{
A Proposed New Model for Denton Proportional Method Generalization in Quarterly Disaggregation of the Gross Domestic Product
}

\author{
Raïmi Aboudou Essessinou ${ }^{1,2, *}$, Guy Degla ${ }^{1}$ \\ ${ }^{1}$ Institute of Mathematics and Physics Sciences (IMSP), University of Abomey-Calavi (UAC), Dangbo, Benin \\ ${ }^{2}$ National Institute of Statistics and Economic Analysis (INSAE), Cotonou, Benin
}

Email address:

raimi.essessinou@imsp-uac.org (R. A. Essessinou),gdegla@imsp-uac.org (G. Degla)

${ }^{*}$ Corresponding author

To cite this article:

Raïmi Aboudou Essessinou, Guy Degla. A Proposed New Model for Denton Proportional Method Generalization in Quarterly Disaggregation of the Gross Domestic Product. Mathematics Letters. Vol. 5, No. 4, 2019, pp. 47-53. doi: 10.11648/j.ml.20190504.12

Received: December 26, 2019; Accepted: January 8, 2020; Published: January 21, 2020

\begin{abstract}
The estimation of a quarterly Gross Domestic Product (GDP) using an indirect approach based on quarterly indicators is often done by quarterly disaggregation of the national accounts branch per branch. The proportional method proposed by Denton (1971) leads to solving several optimization programs and doesn't take into account the links between branches when searching for a quarterly GDP through indirect approach. This paper proposes a brand new approach to quarterly disaggregation of a GDP broken down into several branches or sectors, that takes into account the links between this branches. This approach generalizes the Denton (1971) proportional method and is an integrated approach in which the quarterly disaggregation of GDP is formulated as a multiobjective optimization problem. In fact, we group together on the one hand, all the elementary objective functions identified with respect to the various branches, and on the other hand, all the prescribed constraints according to the branches. Our technique relies on the minimization (in the least squares sense) of the difference in adjustment from neighbouring quarters under constraints provided by the annual benchmark data. The proposed model can be applied to the national accounts of any country using any approach for GDP compilation and it also takes also into account the usual inter dependence of its branches lacking in Denton method. Besides, it is worth noting that an algorithmic method needs to be devised due to the large scale of concrete GDP disaggregation problems.
\end{abstract}

Keywords: National Accounts Disaggregation, Gross Domestic Product Quarterly Disaggregation,

Denton Proportional Method, Generalized Denton Proportional Method, Multiobjective Quadratic Programming

\section{Introduction}

The estimation of quarterly GDP using an indirect approach based on quarterly indicators is often done by quarterly disaggregation of the national accounts for the various branches that make up GDP. This approach leads to the resolution of several optimization programs. The basic idea quarterly disaggregation of national accounts based on an indirect approach with indicators is to "adapt" the indicator to the annual accounts, estimating the statistical relationship that links in the past, the annualized indicator to the corresponding account, and assuming that this relationship observed on annual data is also valid on quarterly data. Among the indirect methods, one of them retains our attention, namely the proportional method of Denton (1971) in [8,9] which is more suitable in case of lack of long time series data. Many working papers used Denton's basic formulation to propose modifications, as have done Cholette and Dagum (1994) in [8], Dagum and Cholette (2006) in [9].

Using the index isomorphism transforming proposed by Essessinou et al. [13], the basic version of Denton's proportional method for the quarterly disaggregation of national accounts is presented as follows $[6,8,9]$ :

$$
\operatorname{Min}_{X_{t}} \sum_{t=2}^{4 T}\left(\frac{X_{t}}{I_{t}}-\frac{X_{t-1}}{I_{t-1}}\right)^{2}
$$


subject to

$$
\sum_{t=4 y-3}^{4 y} X_{t}=A_{y} \text { for } \mathrm{y}=1,2, \ldots, \mathrm{T}
$$

where

$y$ : is the generic index for the annual national account series,

$t$ : is the generic index of quarters on the $T$ years' period: the indexes $i$ and $y$ allowing to reference an observation are restated using an operator proposed in [13],

$A_{y}$ : is the annual national account series for year $y$,

$X_{t}$ : is the desired quarterly value of the national account for the quarter $t$,

$I_{t}$ : is the level of quarterly indicator related to the national account,

$T$ : is the number of year available for national annual account series.

This technique keeps the calibrated series as proportional to the indicator as possible by minimizing (in the sense of least squares) the difference in adjustment from neighbouring quarters under constraints provided by the annual benchmark data. Denton's proportional method is a two-step technique for adjusting the quarterly series: preliminary estimation and adjustment to meet annual constraints. This method is called proportional because it assumes that there is a proportionality relationship between the value of the quarterly account and that of the related indicator.

The multiobjective analysis carried out is based on the fact that there is an interdependence between different branches of the national accounts. It is assumed that there is perfect additivity between sectoral residuals in the determination of the quarterly GDP values. Indeed, certain branches can influence each other. For example, the agriculture branch is linked to other branches of national accounts, namely: agri-food, industry, trade, transport, etc. The quarterly agricultural production can therefore influence not only certain branches but also the analysis of production in certain sectors in conjunction with other sectors of the economy. So, on retrospective data, the quarterly disaggregation of national accounts, based on branch-by-branch disaggregation for the determination of quarterly GDP, does not take into account the links between branches of national accounts in the production process.

In addition, in the classical and traditional methods of annual national accounts decomposition, the links between the branches are not explicitly formalized in the quarterly gross domestic product. Even if the quarterly GDP can be obtained by disaggregating the national accounts of the branches or sectors, using the various existing methods, multivariate approaches including those of Denton and Di Fonzo, T. and M. Marini summarized in the manual of quarterly national accounts [9] and that presented in the works of Ana Abad [2], do not take into account the interactions between branches.

This paper presents a brand new approach for quarterly disaggregating of GDP broken down into several branches or sectors. The proposed model takes into account the links between branches and generalizes the Denton proportional method.

\section{Methodology of the Theoretical Model}

First of all, it is necessary to recall the notion of a multiobjective optimization problem.

Definition 1

Let $\mathrm{U}$ be a nonempty set in $\mathbb{R}^{N}$ and let $\mathrm{F}$ be a vector-valued function from $\mathbb{R}^{N}$ to $\mathbb{R}^{Q}$. The space $\mathbb{R}^{Q}$ is assumed to be partially ordered by a convex and pointed cone C. Please, see [14] for more details on convex and pointed cone.

A multiobjective programming problem is generally formulated as follows:

$$
\operatorname{Min}_{x \in U} F(x)=\left(f_{1}(x), f_{2}(x), \ldots, f_{Q}(x)\right)
$$

where $F: \mathbb{R}^{N} \rightarrow \mathbb{R}^{Q}$ is the vector valued objective function and $U \subseteq \mathbb{R}^{N}$ the set of all the equality and inequality constraints.

A solution $x$ is a vector of $N$ decision variables: $x=$ $\left(x_{1}, x_{2}, \ldots x_{N}\right), x \in \mathbb{R}^{N}$. Each decision variable $x_{i}$ is framed by a lower bound $x_{l i}$, and an upper bound $x_{u i} . f_{i}$ represents the $q^{\text {th }}$ objective function. $Q$ is the number of objective functions and generally we have $Q \geq 2$.

Definition 2 (MOPTD)

By grouping together all the elementary objective functions identified for all branches, and the constraints identified, the quarterly disaggregation of GDP appears as a problem formulated in the form of multiobjective programming. Thus, the multiobjective programming temporal disaggregation (MOPTD) model proposed in this paper is presented in the program defined through the equations (4)-(9) as follows:

$$
\operatorname{Min}_{X}\left\{\left(f_{1}(X), f_{2}(X), \ldots, f_{k}(X), \ldots, f_{M}(X)\right)\right\}
$$

subject to.

$$
\begin{gathered}
X=\left(X_{1}, X_{2}, X_{3}, \ldots . X_{M}\right) \\
X_{k}=\left(X_{k, t}\right)_{t} ; t=1,2, \ldots, 4 T ; \forall k=1,2, \ldots, M \\
-X_{k, t} \leq 0 ; \forall t=1,2, \ldots, 4 T ; \forall k \\
\sum_{t=4 y-3}^{4 y} X_{k, t}-Z_{k, y}=0 ; \forall y=1,2, \ldots, T ; \forall k \\
\sum_{t=4 y-3}^{4 y} \frac{X_{k, t}}{I_{k, t}} \eta_{k, t}=\frac{z_{k, y}}{\sum_{t=4 y-3}^{4 y} I_{k, t}} ; \forall y ; \forall k
\end{gathered}
$$

where all objective functions, constraints, variable and parameters are described as following:

$M$ : the number of branches of national accounts, corresponding to the number of objective functions,

$T$ : the number of years for national accounts observed,

$y \in\{1,2,3, \ldots, T\}$, year generic index,

$i \in\{1,2,3,4\}$, quarter index,

$t \in\{1,2,3, \ldots, 4 T\}$ : generic index of quarters on the $T$ years' period obtained from $i$ and $y$ a using an operator proposed in [13]:

$k \in\{1,2,3, \ldots, M\}$, generic branch index of the quarterly accounts nomenclature.

Value of the annual account per branch: let $Z_{k, y}$ be the (known) value added of the branch account $k$, for the year $y$; 
$I_{k, t}$ is the quarterly indicator value of the branch $k$, for the quarter $t=1,2,3, \ldots ., 4 T$;

$I_{k}=\left(I_{k, t}\right)_{t=1,2, . .4 T}$ : the vector of quarterly indicator related the branch $k$ over the entire period;

Inter-branch interaction: we note $\bar{W}_{j, k}$ the interaction of the branch $j$ on the branch $k$, considered as the average share of the branch $k$ demand of product coming from the branch $j$ :

$$
\bar{W}_{j, k}=1 \text { if } j=k \text { and } 0 \leq \bar{W}_{j, k}<1 \text { if } j \neq k ;
$$

Value added per branch: let $X_{k, t}$ be the value of national account for branch $k$ at quarter

$$
t=1,2,3, \ldots, 4 T ;
$$

The vector of national account value for branch $k$ over the entire period is noted:

$$
X_{k}=\left(X_{k, t}\right)_{t=1,2, . .4 T}, X_{k} \in \mathbb{R}^{4 T} \text { for } k=1, \ldots, M ;
$$

The vector of quarterly national accounts value for all branches over the entire period is noted:

$$
X=\left(X_{1}, X_{2}, X_{3}, \ldots . X_{M}\right), X \in \mathbb{R}^{4 T \times M} ;
$$

Quarterly Gross Domestic Product for the quarter $t: G D P_{t}$ Thus, the objective functions are given by:

$$
\begin{gathered}
f_{k}\left(X_{1}, X_{2}, \ldots, X_{M}\right)=\sum_{j=1}^{M} \sum_{t=2}^{4 T} \bar{W}_{j, k}\left(\frac{X_{j, t}}{I_{j, t}}-\frac{X_{j, t-1}}{I_{j, t-1}}\right)^{2} \\
\text { for } k=1,2, \ldots, M
\end{gathered}
$$

The quarterly Gross Domestic Product is expressed by the relationship:

$$
G D P_{t}=\sum_{\mathrm{k}=1}^{\mathrm{M}} X_{k, t} ; \text { for } t=1,2, \ldots, 4 T
$$

Annual Gross Domestic Product for the year is obtained by aggregation over the four quarters

$$
G D P_{y}=\sum_{\mathrm{t}=4 \mathrm{y}-3}^{4 \mathrm{y}} G D P_{t} ; \text { for } y=1,2, \ldots, T
$$

\section{Proposition 1}

Under the assumption of a total absence of interactions between branches, the program presented through the equations (4)-(9) is reduced to the Denton proportional method applied to each of the $M$ branches of national accounts.

Proof

Let suppose that there is no interaction between the $M$ branches of national accounts.

So we have $\overline{\mathrm{W}}_{\mathrm{j}, \mathrm{k}}=1$ if $j=k$ et $\overline{\mathrm{W}}_{\mathrm{j}, \mathrm{k}}=0$ if $j \neq k$; $k=1,2,3, \ldots, M$

Consequently, $f_{k}$ becomes

$$
f_{k}(X)=\sum_{t=2}^{4 T}\left(\frac{\mathrm{X}_{\mathrm{k}, \mathrm{t}}}{\mathrm{I}_{\mathrm{k}, \mathrm{t}}}-\frac{\mathrm{X}_{\mathrm{k}, \mathrm{t}-1}}{\mathrm{I}_{\mathrm{k}, \mathrm{t}-1}}\right)^{2} ;
$$

$$
\forall k=1,2, \ldots, M \text { with } X=\left(X_{1}, X_{2}, X_{3}, \ldots, X_{M}\right)
$$

$f_{k}(X)$ therefore depends only on $X_{\mathrm{k}}, \forall k=1,2, \ldots, M$.
The problem defined by the equations (4)-(9) can therefore be written as follows:

$$
\operatorname{Min}_{\mathrm{X}}\left\{\left(f_{1}\left(X_{1}\right), \ldots, f_{k}\left(X_{\mathrm{k}}\right), \ldots, f_{M}\left(X_{\mathrm{M}}\right)\right)\right\}
$$

subject to

$$
\left\{\begin{array}{c}
X=\left(X_{1}, X_{2}, X_{3}, \ldots . X_{M}\right) \\
X_{k}=\left(X_{k, t}\right)_{t} ; t=1,2, \ldots, 4 T ; k=1,2, \ldots, M \\
-X_{k, t} \leq 0 ; \forall t=1,2, \ldots, 4 T ; k=1,2, \ldots, M \\
\sum_{t=4 y-3}^{4 y} X_{k, t}-Z_{k, y}=0 ; \forall y=1,2, \ldots, T ; \forall k \\
\sum_{t=4 y-3}^{4 y} \frac{X_{k, t}}{I_{k, t}} w_{k, t}=\frac{Z_{k, y}}{\sum_{t=4 y-3}^{4 y} I_{k, t}} ; \forall k ; \forall y
\end{array}\right.
$$

By relaxing the constraint

$$
\sum_{t=4 y-3}^{4 y} \frac{X_{k, t}}{I_{k, t}} w_{k, t}=\frac{z_{k, y}}{\sum_{t=4 y-3}^{4 y} I_{k, t}} ; k=1, \ldots, M ; \forall y=1, \ldots, T,
$$

we get the following new program presented in (14):

$$
\operatorname{Min}_{X}\left\{\left(f_{1}\left(X_{1}\right), \ldots, f_{k}\left(X_{\mathrm{k}}\right), \ldots, f_{M}\left(X_{\mathrm{M}}\right)\right)\right\}
$$

subject to

$$
\left\{\begin{array}{c}
X=\left(X_{1}, X_{2}, X_{3}, \ldots, X_{M}\right) \\
X_{k}=\left(X_{k, t}\right)_{t} ; t=1,2, \ldots, 4 T ; \forall k=1,2, \ldots, M \\
-X_{k, t} \leq 0 ; \forall t=1,2, \ldots, 4 T ; \forall k \\
\sum_{t=4 y-3}^{4 y} X_{k, t}-Z_{k, y}=0 ; \forall y=1,2, \ldots, T ; \forall k M
\end{array}\right.
$$

Since the objective functions have independent arguments, it is possible to optimize the $f_{k}$ separately to determine the $X_{k}=\left(X_{k, t}\right)_{t}$ for $k=1,2, \ldots, M$, so that we can have the $G D P_{t}$ by summing the $X_{k, t}$, using the equation (11). This means solving all the elementary optimization program defined by the equation (15):

$$
\operatorname{Min}_{X_{k}}\left\{f_{k}\left(X_{\mathrm{k}}\right)\right\}
$$

subject to

$$
\begin{gathered}
-X_{k, t} \leq 0 ; \forall t=1,2, \ldots, 4 T ; \\
\sum_{t=4 y-3}^{4 y} X_{k, t}-Z_{k, y}=0 ; \forall y=1,2, \ldots, T
\end{gathered}
$$

It can be seen that the program presented in (15) is similar to the program presented earlier in (1), illustrating the Denton proportional method applied to branch $k=1,2, \ldots, M$ of national accounts.

Thus, the program presented through the equations (4)-(9) is reduced to Denton's proportional method applied successively to the $M$ branches to deduct quarterly GDP from the previous equation (11).

Remark 1

From the results of proposition 1, it can be deduced that the proposed MOPTD model is a generalization of Denton's proportional method. 


\section{Theoretical Existence of Solution}

The problem is analyzed on the basis of a hypothesis of cooperation between the objective functions. The following definition provides an understanding of concepts and mathematical properties of the proposed model.

Definition 3

Given $n$ variables $x_{1}, x_{2}, x_{3}, \ldots, x_{n}$ and the subset $K$ of $\mathbb{R}$, we call quadratic polynomial in $x_{1}, x_{2}, x_{3}, \ldots, x_{n}$, any application $\varphi: K^{n} \rightarrow K$ such that there are elements $\alpha_{i i}(1 \leq i \leq n)$ and $\alpha_{i j}(1 \leq i<j \leq n)$ of the $K$ and:

$$
\varphi\left(x_{1}, x_{2}, x_{3}, \ldots, x_{n}\right)=\sum_{i=1}^{n} \alpha_{i i} x_{i}^{2}+2 \sum_{1 \leq i<j \leq n} \alpha_{i j} x_{i} x_{j}
$$

Definition 4 [10]

(i) A function $f: \mathrm{K} \rightarrow \mathbb{R}$ is said to be quadratic if $f$ is the sum of an affine function and a quadratic form. That is to say

$$
f(X)=<A X, X>+<C, X>
$$

where $\mathrm{K} \subset \mathbb{R}^{n} ; \mathrm{A}$ is a square matrix of $M_{n}(\mathbb{R})$ and $\mathrm{C}$ is a matrix of size $1 \times n$.

(ii) An optimization problem (P) is quadratic if the criterion is quadratic and has a finite number of linear constraints of equality and inequality. In other words, (P) is the form presented by the equation (18):

$$
\operatorname{Min} f(X)=<A X, X>+<C, X>
$$

s. $\mathrm{t}$

$$
\left\{\begin{array}{l}
B X=b \\
D X \leq d
\end{array}\right.
$$

A is a square matrix of $M_{n}(\mathbb{R})$ and $\mathrm{C}$ is a matrix of size $1 \times n$; b is a matrix of size $\mathrm{k} \times 1$; $\mathrm{d}$ is a matrix of size $\mathrm{m} \times 1$; $\mathrm{B}$ is a matrix of $M_{k \times n}(\mathbb{R})$ and $\mathrm{D}$ is a matrix of $M_{m \times n}(\mathbb{R})$.

Remark 2

Obviously, any quadratic function is polynomial and therefore continuous.

Definition 5

An optimization problem is said to have a finite value, if its set of solutions is not empty and its objective function is to have real values is defined by: $F: U \rightarrow \mathbb{R}$; where $U$ is a normed vector space.

We have the following existence theorem.

Theorem 1

If $\Omega$ is a normed vector space with finite dimension, then any finite value quadratic optimization problem on $\Omega$ admits (at least) a solution.

Proof

This theorem is a special case of Theorem 1.36 in [10] formulated in the case where $\Omega$ is a Banach space

Theorem 2 ([14], p 274)

Assume that $\operatorname{cl}(C)$ is pointed, $U$ is a nonempty compact set, and $F$ is a continuous function. Then, the problem (3) has minimal solutions.

\section{Proof}

This theorem is an equivalent version case of Theorem 7.5 for minimization programming in [14]. The proof can be seen in [14].

\section{Definition 6}

Considering the objective function $f_{k}$ defined in equation (10), the elementary problem of quarterly disaggregation of branch (sector) $k$ is presented through the equations (19)-(24) as follows:

$$
\operatorname{Min}_{X}\left\{f_{k}(X)\right\}
$$

subject to

$$
\begin{gathered}
X_{k}=\left(X_{k, t}\right)_{t} ; t=1,2, \ldots, 4 T \\
X=\left(X_{1}, X_{2}, X_{3}, \ldots . X_{N}\right) \\
-X_{k, t} \leq 0 ; \forall t=1,2, \ldots, 4 T \\
\sum_{t=4 y-3}^{4 y} X_{k, t}-Z_{k, y}=0 ; \forall y=1,2, \ldots, T \\
\sum_{t=4 y-3}^{4 y} \frac{X_{k, t}}{I_{k, t}} w_{k, t}=\frac{z_{k, y}}{\sum_{t=4 y-3}^{4 y} I_{k, t}} ; \forall y=1,2, \ldots, T
\end{gathered}
$$

Remark 3

As it can be seen, this problem is multiobjective quadratic programming.

Lemma 1

If $\mathrm{T}$ is finite number, then for all $k=1,2, \ldots, M$, the problem is presented through the equations (19)-(24) is quadratic with a compact constrained set and therefore admits a solution.

Proof

Let start by noting that the objective function $f_{k}$ defined in equation (10) is a quadratic polynomial according to the definition 3 .

Indeed, for fixed $k$, by posing $\mathrm{a}_{\mathrm{j}, \mathrm{t}}=\frac{1}{\mathrm{I}_{\mathrm{j}, \mathrm{t}}}$ we have:

$$
f_{k}(X)=\sum_{j=1}^{M} \sum_{t=2}^{4 T} \bar{W}_{j, k}\left(\mathrm{a}_{\mathrm{j}, \mathrm{t}} X_{j, t}-\mathrm{a}_{\mathrm{j}, \mathrm{t}-1} X_{j, t-1}\right)^{2}
$$

$=\sum_{j=1}^{M} \sum_{t=2}^{4 T} \bar{W}_{j, k}\left(\mathrm{a}_{\mathrm{j}, \mathrm{t}}^{2} X_{j, t}^{2}+\mathrm{a}_{\mathrm{j}, \mathrm{t}-1}^{2} X_{j, t-1}^{2}-2 a_{\mathrm{j}, \mathrm{t}} a_{\mathrm{j}, \mathrm{t}-1} X_{j, t} X_{j, t-1}\right)$

by developing and reducing relative to $t$, we obtain;

$$
\begin{gathered}
f_{k}\left(X_{1}, X_{2}, \ldots, X_{M}\right)= \\
\sum_{j=1}^{M} \bar{W}_{j, k}\left(\mathrm{a}_{\mathrm{j}, 1}^{2} X_{j, 1}^{2}+2 \mathrm{a}_{\mathrm{j}, 2}^{2} X_{j, 2}^{2}+\cdots+2 \mathrm{a}_{\mathrm{j}, 4 \mathrm{~T}-1}^{2} X_{j, 4 \mathrm{~T}-1}^{2}\right. \\
\left.+\mathrm{a}_{\mathrm{j}, 4 \mathrm{~T}}^{2} X_{j, 4 \mathrm{~T}}^{2}\right) \\
+2 \sum_{j=1}^{M} \bar{W}_{j, k}\left(-a_{\mathrm{j}, 1} a_{\mathrm{j}, 2} X_{\mathrm{j}, 1} X_{\mathrm{j}, 2}-a_{\mathrm{j}, 2} a_{\mathrm{j}, 3} X_{\mathrm{j}, 2} X_{\mathrm{j}, 3}-\cdots\right. \\
\left.-a_{\mathrm{j}, 4 \mathrm{~T}-1} a_{\mathrm{j}, 4 \mathrm{~T}} X_{j, 4 \mathrm{~T}-1} X_{j, 4 \mathrm{~T}}\right)
\end{gathered}
$$

by posing $\alpha_{1,1, j}=\mathrm{a}_{\mathrm{j}, 1}^{2} \bar{W}_{j, k} ; \alpha_{4 T, 4 T, j}=\mathrm{a}_{\mathrm{j}, 4 \mathrm{~T}}^{2} \bar{W}_{M, k} ; \alpha_{l, l, j}=$ $2 \mathrm{a}_{\mathrm{j}, \mathrm{i}}^{2} \bar{W}_{M, k}, l=2,3, \ldots 4 T-1$ et

$$
\alpha_{i, l, j}=-a_{\mathrm{j}, \mathrm{i}} a_{\mathrm{j}, \mathrm{l}} \overline{\mathrm{W}}_{\mathrm{j}, \mathrm{k}}(1 \leq i<l \leq 4 T)
$$


we obtain

$$
\begin{gathered}
f_{k}\left(X_{1}, X_{2}, \ldots, X_{M}\right)= \\
\sum_{j=1}^{M}\left(\alpha_{1,1, j} X_{j, 1}^{2}+\alpha_{2,2, j} X_{j, 2}^{2}+\cdots+\alpha_{4 T-1,4 T-1, j} X_{j, 4 \mathrm{~T}-1}^{2}\right. \\
\left.+\alpha_{4 T, 4 T, j} X_{j, 4 \mathrm{~T}}^{2}\right) \\
+2 \sum_{j=1}^{M}\left(\alpha_{12, j} X_{j, 1} X_{j, 2}+\alpha_{23, j} X_{j, 2} X_{j, 3}+\cdots\right. \\
\left.+\alpha_{4 T-1,4 T, j} X_{j, 4 \mathrm{~T}-1} X_{j, 4 \mathrm{~T}}\right)
\end{gathered}
$$

Thus $f_{k}$ is a quadratic polynomial.

Let us designate by $\Omega_{k}$ the constrained space of the problem presented through the equations (19)-(24).

we have:

$$
\begin{gathered}
\Omega_{k}=\left\{X_{k}=\left(X_{k, t}\right)_{t} ; \forall y=1,2, \ldots, T ; t=1,2, \ldots, 4 T ;\right. \\
-X_{k, t} \leq 0 ; X_{k, t}-Z_{k, y} \leq 0 ; \\
\sum_{t=4 y-3}^{4 y} X_{k, t}-Z_{k, y}=0 ; \\
\left.\sum_{t=4 y-3}^{4 y} \frac{X_{k, t}}{I_{k, t}} w_{k, t}=\frac{Z_{k, y}}{\sum_{t=4 y-3}^{4 y} I_{k, t}}\right\}
\end{gathered}
$$

$\Omega_{k}$ consists of the constraints of equality and inequality defined by affine functions. Thus, as $f_{k}$ is a quadratic polynomial, according to definition 4 (ii), the problem presented through the equations (19)-(24) is quadratic. In addition to that $f_{k}$ is continuous over $\Omega_{k}$ according to the remark 3. As $\Omega_{k}$ is a normed vector sub-space of $\mathbb{R}^{4 T}$, if T is finite, then $\Omega_{k}$ is of finite size. In addition, as $Z_{k, y}$ is deterministic because being a fixed base data, $\Omega_{k}$ is closed and bounded. Thus, applying Theorem 2, we deduce that the problem presented through the equations (19)-(24) admits at least one solution.

Theorem 3

Let us $\Omega_{k}$ be the constraints space of the elementary problem presented through the equations (19)-(24). So, if T is finite, the problem presented through the equations (4)-(9) admits at least one solution.

Proof

We assume that $\mathrm{T}$ is finite. According to Lemma 1, we have the following results for all $k$ :

a) the objective function $f_{k}$ is quadratic,

b) $\Omega_{k}$, is part of a vector space of finite dimension, bounded and

c) the problem presented through the equations (19)-(24) is quadratic and has a solution.

We therefore deduce that $\forall k=1,2, \ldots, M, f_{k}$ is regular, i.e. continuous, finite and bounded on $\Omega_{k}$.

Let consider $\Lambda=\Gamma \cap\left(\prod_{k} \Omega_{k}\right)$ where $\Gamma$ is the vector space defined by:

$$
\begin{gathered}
\Gamma=\left\{\left(X_{k}\right)_{k=1,2, \ldots, M}=\left(\left(X_{k, t}\right)_{t}\right)_{k=1,2, \ldots, M}, t=1,2, \ldots, 4 T ;\right. \\
\text { with } \left.\sum_{t=4 y-3}^{4 y} \sum_{\mathrm{k}=1}^{\mathrm{N}} X_{k, t}-\sum_{k=1}^{N} Z_{k, y}=0 ; \forall y=1,2, \ldots, T\right\}
\end{gathered}
$$

By construction, $\Gamma$ is made up of equality constraints defined by affine functions. $\Gamma$ explain that for each year, the total values added of the four quarters for all the branches (sectors) is equal to the GDP, this balance is realized over the entire period of the annual accounts observation.

Thus, we have $\Gamma \subset\left(\mathbb{R}^{4 T}\right)^{M} \equiv \mathbb{R}^{4 T M}$ and we obtain that $\Gamma$ is an affine sub-space of $\mathbb{R}^{4 T M}$ which is of finite size.

As $\Omega_{k}$ is of finite dimension and is bounded, so $\prod_{k} \Omega_{k} \subset$ $\left(\mathbb{R}^{4 T}\right)^{M}$ is of finite and bounded dimension. Consequently, $\Lambda=\Gamma \cap\left(\prod_{k} \Omega_{k}\right)$ is of finite dimension, bounded and therefore constitutes a regular space.

Let's $\Sigma$ be the space defined by all the constraints of the problem presented through the equations (4)-(9). Intuitively, the multiobjective program which is defined reflects that $\Sigma \subseteq \Lambda$. This implies that $\Sigma$ is a regular space.

Thus, the regularity properties of objective functions and the characteristics of the spaces of decision vectors built from constraints, illustrate the regularity of the problem. The existence of a solution is therefore ensured by Theorem 2 above and the Theorem 1 of [3] in the case of one level.

Remark 4

Denton's proportional method implicitly establishes from the observed annual benchmark indicator (BI) ratio a time series of estimation-indicator ratios (quarterly BI ratio) of quarterly estimates calibrated from the quarterly national accounts that is as smooth as possible and such that, in the case of flow series:

(a)- For retrospective series $(y \in\{1,2, \ldots, T\})$, the average of the quarterly $\mathrm{BI}$ ratio is equal to the annual $\mathrm{BI}$ ratio for each year $y$.

(b)- For prospective series $(y \in\{T+1, \ldots\})$, quarterly ratios are kept constant and equal to the ratio of the last quarter of the last benchmark year.

The Remark 4 is used to reinforce constraints and to develop an improved version of adjustment and extrapolation method in the multi-objective approach we propose in this paper. So we have the following definitions.

Definition 7 (Adjusting)

To reconcile the estimated quarterly national accounts with the true annual observed values, an adjustment is proposed to the estimated quarterly values. The adjusted value $X_{k, t}^{\text {Adj }}$ of the estimated national accounts for quarter $t$ for branch (sector) $k$ is given by:

$$
X_{k, t}^{A d j}=\hat{X}_{k, t}-\eta_{k, t} \times A b s\left(\sum_{t=4 y-3}^{4 y} \hat{X}_{k, t}-Z_{k, y}\right)
$$




$$
\begin{gathered}
\times \operatorname{sign}\left(\sum_{t=4 y-3}^{4 y} \hat{X}_{k, t}-Z_{k, y}\right) \\
\forall y=1,2, \ldots . T
\end{gathered}
$$

The weights are given by:

$$
\eta_{k, t}=\frac{I_{k, t}}{\sum_{t=4 y-3}^{4 y} I_{k, t}}
$$

$\hat{X}_{k, t}$ is the estimated value of the account for the quarter and $Z_{k, y}$ the value for the year $y$

$\operatorname{Abs}(x)$ is the absolute value of $x$ :

$$
\operatorname{Abs}(x)=\max \{-x, x\}
$$

$$
X_{k, 4 m+r}^{A d j}=I_{k, 4 m+r}\left[0.5 \times \frac{X_{k, 4 m+r-1}^{A d j}}{I_{k, 4 m+r-1}}+0.5 \times\left(\frac{1}{9} \sum_{t=(4 m+r-1)-9}^{(4 m+r-1)} \frac{X_{k, t}^{A d j}}{I_{k, t}}\right)\right]
$$

where

$X_{k, t}^{A d j}$ is the adjusted interpolation value added of the branch $k$ for the quarter $t$

$r$ is the rank of the quarter of year $m+1$ for which the extrapolation is made, $r=1,2,3,4$.

\section{Numerical Algorithm in Investigation}

The solving algorithms of the traditional quarterly disaggregation methods are presented in $[1,2,5]$. The solving method and algorithm of the model presented in this paper are in progress.

Several algorithms have been developed in the literature to solve multiobjective optimization problems, but most of these multiobjective optimization problems are Non Polynomial difficult (NP-difficult) problems. In the class of NP-difficult problems, there is no algorithm that provides the optimal solution in polynomial time depending on the size of the problem. Solving multiobjective problems comes from two fairly different disciplines. Two types of approaches are adopted, namely: non-Pareto approaches which seek to reduce the initial problem to one or more mono-objective problems and Pareto approaches which do not transform the objectives of the problem and these are treated simultaneously during the solving by seeking the best compromise solutions.

The challenge that we faced in this research is that of the search for an algorithm allowing to have a satisfactory result especially in a context where the economic activity takes place in a continuous and dynamic way with interaction between the branches of the economy. The model solving could be done following the Pareto approach by relying on Non-dominant Sorting Genetic Algorithm (NSGA-II) [4, 15] procedure. However, NSAG-II introduces a hazard when generating the reference population for Pareto optimal solutions searching.

Given that, it is necessary to set up a method to make the fairest or most desirable decision [11], the first phase of the next research consists in setting up a method and an algorithm allowing to solve in a real way the proposed model in order to get strictly the point which achieves the minimum of each objective function.

\section{Conclusion and Perspective}

This paper provides a theoretical presentation of the problem of quarterly disaggregation of GDP in the form of a multiobjective programming model. It should be noted that the proposed model in this paper is better suited for the quarterly disaggregation of GDP. The MOPTD model we propose generalizes the Denton's proportional method.

Finally, this paper shows that the extrapolations of GDP using the Denton proportional method and those made using the multiobjective programming approach we propose, must produce similar results when the quarterly indicators used are strongly correlated to the annual accounts. It should be noted that the multiobjective programming approach is better suited to take into account the links between branches of national accounts in the GDP quarterly disaggregation process. Our approach could reduce the volatility of the quarterly GDP obtained. The proposed model can be applied to the national accounts of any country using any of (i) production approach, (ii) expenditure approach and (iii) income approach for GDP compilation.

The problem is in evidence a large problem and work is in progress to set up an efficient method and algorithm for solving our model. Simulations will be carried out using the real data of a National Statistics Office (NSO). The data will subject to prior statistical processing before proceeding to the simulations. The GDP will be decomposed into several branches in accordance with the nomenclature defined for the national accounts at the NSO. The obtained results with the proposed model will be compared to those obtained using Denton's proportional method applied separately to each of the sectors.

\section{Acknowledgements}

We would like to thank all the reviewers of this paper, all 
the authors whose works are used during this research and all those whose observations and comments helped to improve the work. Finally, we would like to send special thanks to the African Centre of Excellence in Mathematical Sciences and Application (ACE-MSA) at Institute of Mathematics and Physics Sciences (IMSP) for all its supports.

\section{References}

[1] Abdelwahed, T., Leila, H.: A Benchmarking Approach for Temporal Disaggregation of Economicc Time Series by Related Series. Working paper and studies, ISSN 1725-4825, ISBN 92-79-01306-8, Luxembourg: Office for Official Publications of the European Communities, 5-10, 29-41 (2005) http://europa.eu.int.

[2] Ana, A., Enrique M. Q.: Software to perform temporal disaggregation of economic time series. Working paper and studies, ISSN 1725-4825, ISBN 92-894-8626-0, Luxembourg: Office for Official Publications of the European Communities, 3-40 (2005). http://europa.eu.int.

[3] Ankur, S., Pekka, M., Kalyanmoy, D.: A Review on Bilevel Optimization: From Classical to Evolutionary Approaches and Applications. arXiv: 1705.06270v1 [math. OC] 17 May 2017, 4-7 (2017).

[4] Aravind, S.: A Fast Elitist Multiobjective Genetic Algorithm: NSGA-II. Mathlab source code (2012). http://www. mathworks $\mathrm{com} / \mathrm{matlabcentral/fileexchange/10429-nsga-iia-multi-objectiv}$ e-optimization-algorithm/content.

[5] Christoph, Sax., Peter, S.: Temporal Disaggregation of Time Series. Contributed research articles, The R Journal Vol. 5/2, December 2013. ISSN 2073-4859, 80-81 (2013), https://journal.r-project.org/archive/2013-2/sax-steiner.pdf.

[6] Di Fonzo, T., Marco, M.: On the Extrapolation with the Denton Proportional Benchmarking Method. IMF Working Paper Series, $\quad \mathrm{WP} / 12 / 169, \quad 5-6 \quad$ (2012), https://www.imf.org/external/pubs/ft/wp/2012/wp12169.pdf.

[7] European Communities, United Nations and al.: System of National Accounts 2008. All rights reserved, New York, 1-7,
39-66, 295-315 (2009),

https://unstats.un.org/unsd/nationalaccount/docs/sna2008.pdf.

[8] Fonds Monétaire International: Manuel des comptes nationaux trimestriels: concepts, sources statistiques et compilation. Compilation de Adriaan M. B., Robert J. D., Nils O. Fonds Monétaire International, Washington, D.C., 93-139 (2001), https://www.imf.org/ /media/Websites/IMF/imported-publicat ions-loe-pdfs/external/pubs/ft/qna/2000/textbook/fra/text.ashx.

[9] International Monetary Fund: Quarterly National Accounts Manual. 2017 Edition, Pre-Publication, november 2018, 86-126, (2018), https://www.imf.org/external/pubs/ft/qna/.

[10] Fréderic, B.: Optimisation continue, Cours et problème corrigé. Sciences Sup, Maths. Appl. pour le Master/SMAI, 327 pages Dunod, Paris, 20-21 (2006).

[11] Julien, C.: Analyse post-Pareto en Optimisation Vectorielle Stochastique et Déterministe: Étude Théorique et Algorithmes. Thèse de doctorat, Mathématiques, Université de la Nouvelle-Calédonie, 19 (2014), https://tel.archives-ouvertes.fr/tel-01337505/document.

[12] Marco M.: Nowcasting Annual National Annual National Accounts with Quarterly Indicateor: An Assessment of Widely Used Benchmarking Methods. IMF Working Paper. WP/16/71, march 2016,

https:/www.imf.org/external/pubs/ft/wp/2016/wp1671.pdf.

[13] Raïmi Aboudou Essessinou, Guy Degla and Babacar Mbaye Ndiaye: From Two to One Index Isomorphism in Optimization Program for Quarterly Disaggregation of Annual Times Series. Journal of Advances in Mathematics and Computer Science, 34 (1 \& 2): 1-15, 2019; Article no. JAMCS.51632, http://www.journaljamcs.com/index.php/JAMCS/article/view/ 30199.

[14] Saleh A. R. Al-Mazel, Falleh R. M. Al-Solamy, Qamrul H. Ansari: Fixed Point Theory, Variational Analysis and Optimization. CRC Press, Taylor and Francis Group, A CHAPMAN \& HALL BOOK, Pages 273-334.

[15] Srinivas N., Kalyanmoy D.: Multiobjective optimization using Nondominated Sorting Genetic Algorithm. Journal of Evo. Comput. Vol. 2, No. 3, Pages 221-248, 7-12 (1994), https://web.njit.edu/ horacio/Math451H/download/SrinivasDe b_GA.pdf. 